\title{
63 High-performance sports and non-specific immunity
}

During strenuous endurance exercises, the secretion of cortisol increases two- to three-fold and then superimposes the effect of the catecholamine release. As a result, the number of NK cells rises up to 9 times the initial value and drops back to $50 \%$ of the initial value for a few hours after exercise has finished. In addition, cytotoxic activity is reduced, regulated by higher prostaglandin F2 levels produced by the monocytes.
Excessive exercise creates an "open window" in the body's immune defenses, making it more vulnerable to pathogens.

Granulocytes behave differently. Their number can increase by up to three times their initial value. Their release from the bone marrow is caused by the cortisol effect. Although they produce $30-50 \%$ fewer reactive oxygen compounds under these conditions as a result of their increased numbers, their bactericidal effect is increased overall. These granulocyte changes are reversed after 1-2 days.

- Fig. 63.1 Cell count changes during intensive endurance workout 